\title{
Status distónico, a propósito de dos casos
}

\author{
Status dystonicus, apropos of two cases
}

Correspondencia

Jose J. Centeno-Arispe

jcentenoarispe@hotmail.com

Recibido: 26/03/2017

Arbitrado por pares

Aprobado: 24/05/2017

Citar como: Centeno-Arispe JJ, Genaro-Saldaña S, Montiel-Blanco JD, Flores-Bravo JC, Caro-Kahn 1. Status distónico, a propósito de dos casos. Acta Med Peru. 2017;34(2):132-5
José J. Centeno-Arispe ${ }^{1, a}$, Susan Genaro-Saldaña $a^{2, b}$, Johnny D. MontielBlanco ${ }^{3, c}$, Julio C. Flores-Bravo ${ }^{3, c}$, Inés Caro-Kahn ${ }^{3, c}$

1 Instituto Nacional de Ciencias Neurológicas. Lima, Perú.

2 Instituto Nacional de Salud del Niño. Lima, Perú.

3 Servicio de Neuropediatría del Instituto Nacional de Salud del Niño. Lima, Perú

a Residente de neurología, b Residente de pediatría, c Neuropediatra

\section{RESUMEN}

El status distónico (SD) es una emergencia médica infrecuente y sub-diagnosticada, común en distonías secundarias; las infecciones son el factor gatillante más frecuente. Puede ser distonía tónica o fásica. El fenotipo tónico es usual en varones, distonías secundarias y tiene peor pronóstico. Se acompaña de hiperpirexia y rabdomiólisis que puede complicarse con falla renal aguda, insuficiencia respiratoria o la muerte. No hay ensayos clínicos disponibles sobre el tratamiento, aunque las benzodiacepinas, trihexifenidilo, levodopa, tetrabenazina, baclofeno, gabapentina han mostrado diversos grados de control sintomático. Se reporta el caso de dos niños, con parálisis cerebral infantil y antecedente de kernícterus que desarrollaron SD después de un cuadro infeccioso, ambos respondieron favorablemente a benzodiacepinas, Levodopa, baclofeno, bromocriptina y gabapentina. En el Perú correspondería a los primeros casos publicados a la fecha.

Palabras clave: Distonía; Rabdomiólisis; Trastornos distónicos (fuente: DeCS BIREME).

\begin{abstract}
Status dystonicus (SD) is a rare and underdiagnosed medical emergency, which is usually triggered by infectious conditions. SD may have tonic or phasic dystony. The tonic phenotype is common in men, and those with secondary dystony may have a worse prognosis. The condition is accompanied by hyperpyrexia and rhabdomyolysis that may complicate with acute renal failure, respiratory insufficiency or even death. There are no clinical trials available with respect to its therapy; but drugs such as benzodiazepines, trihexyphenidyl, levodopa, tetrabenazine, baclofen, and gabapentin have shown different degrees of symptomatic control. This article reports two cases of SD in children with cerebral palsy and a past medical history of kernicterus who developed SD after an infectious condition. Both children responded satisfactorily to benzodiazepines, levodopa, baclofen, bromocriptine, and gabapentin. These are the very first cases of SD reported in Peru.
\end{abstract}

Keywords: Dystonia; Rhabdomyolysis; Dystonic disorders (source: MeSH NLM). 


\section{INTRODUCCIÓN}

El estado distónico (SD) es una emergencia médica infrecuente y subdiagnosticada, definido como "el incremento de la frecuencia y severidad de episodios de distonía generalizada, que necesita ingreso hospitalario urgente" ${ }^{[1,2]}$. Es común en varones y menores de 15 años. En el 67,4\% de los casos se puede identificar el factor desencadenante, siendo el más común las infecciones ${ }^{[3]}$.

EI SD puede ser por distonía tónica (incremento de la intensidad) o fásica (aumento en la frecuencia). El primero es usual en varones y tiene peor pronóstico. Ambos causan hiperpirexia y rabdomiólisis que puede complicarse con falla renal aguda, insuficiencias respiratorias o la muerte (en $10 \%$ de los casos) ${ }^{[4]}$. Se utiliza la escala de severidad de distonía (ESD) propuesta por Lumsden para estratificarlo en cinco grados ${ }^{[5]}$.

No hay ensayos clínicos disponibles sobre la mejor estrategia terapéutica. Aunque las benzodiacepinas, trihexifenidilo, levodopa, tetrabenazina, baclofeno, gabapentina han mostrado diversos grados de control sintomático ${ }^{[4,6]}$.

Reportamos el caso de dos niños, con parálisis cerebral infantil $(\mathrm{PCl})$ y antecedente de kernícterus que desarrollaron SD después de un cuadro infeccioso, ambos respondieron favorablemente a benzodiacepinas, levodopa, baclofeno, bromocriptina y gabapentina. En el Perú correspondería a los primeros casos publicados a la fecha.

\section{REPORTES DE CASOS}

\section{CASO 1}

Niño de 3 años, con antecedente de kernícterus y PCl distónicoespástica. Presenta reiterados ingresos hospitalarios por neumonía aspirativa y reflujo gastroesofágico, medicado regularmente el último año con omeprazol, clonazepam y domperidona. Previamente tolera la sedestación y puede conciliar el sueño ininterrumpidamente durante la noche (Grado 1-ESD). Ocho días antes del internamiento cursa con infección de vías aéreas superiores, recibe antipiréticos y antibióticos orales, cuadro infeccioso remite pero persiste hipertermia, irritabilidad, disturbios en el sueño e incremento de la distonía. Al examen físico se constata hipertermia $\left(40^{\circ} \mathrm{C}\right)$, irritabilidad, marcada distonía generalizada sostenida a predominio de hemicuerpo derecho (Grado 4-ESD), sin evidencia de infección activa. La CPK-CK y transaminasas están elevadas, creatinina sérica es normal. Se manejó inicialmente con midazolam en bolos, las primeras 48 horas. Se continuó con diazepam parenteral, luego bromocriptina, levodopa/ carbidopa y baclofeno. Buena respuesta clínica (Figura 1), con disminución de la hiperpirexia a partir del día 8, los niveles de CPK-CK disminuyen progresivamente, manteniendo una función renal adecuada y egresa con un grado 2 -ESD.

\section{CASO 2}

Varón de 4 años, con antecedente de $\mathrm{PCl}$, kernícterus e ingresos frecuentes por neumonía. Habitualmente adopta una postura distónica generalizada, que no interrumpe el sueño nocturno y se mantiene estable con la medicación recibida (Grado 1-ESD). Siete días previos al ingreso desarrolla neumonía aspirativa que inicialmente remite con antibióticos parenterales, la hipertermia $\left(40-41^{\circ} \mathrm{C}\right)$ no cede con antipiréticos comunes, se incrementa distres respiratorio y distonía sostenida casi todo el día, llegando al opistótonos, que interrumpe el sueño (Grado 4-ESD). Al examen físico tiene tiraje supraclavicular e intercostal, distonía generalizada marcada. La CPK-CK y transaminasas elevadas, creatinina sérica normal. Debido al compromiso respiratorio recibe oxigeno suplementario, se amplía el espectro de antibióticos y se optimiza el manejo de secreciones bronquiales. Se inicia el manejo del SD con baclofeno, diazepam y luego midazolam en infusión, además de

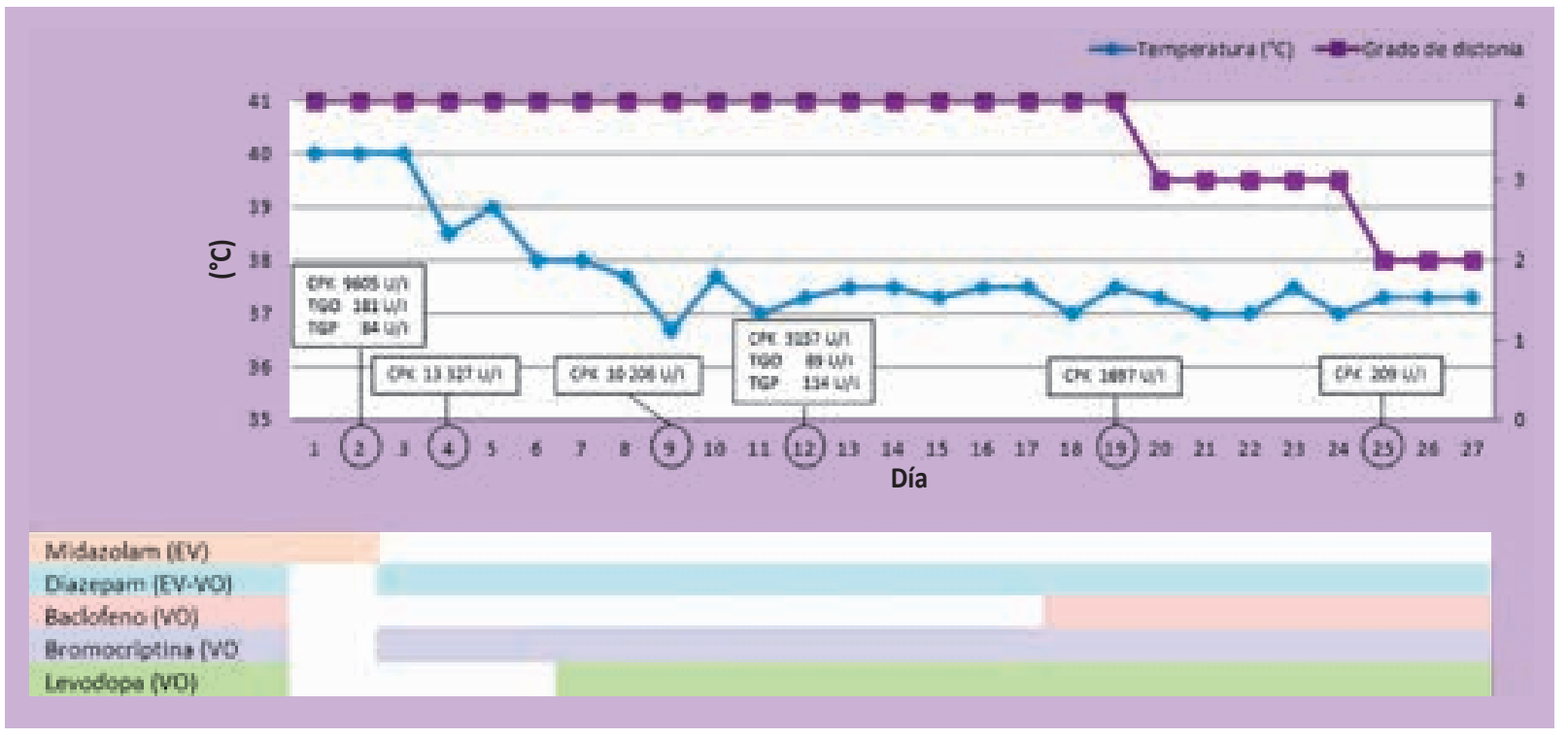

Figura 1. Caso 1, correlación clínica y laboratorial con tratamiento farmacológico instaurado. 


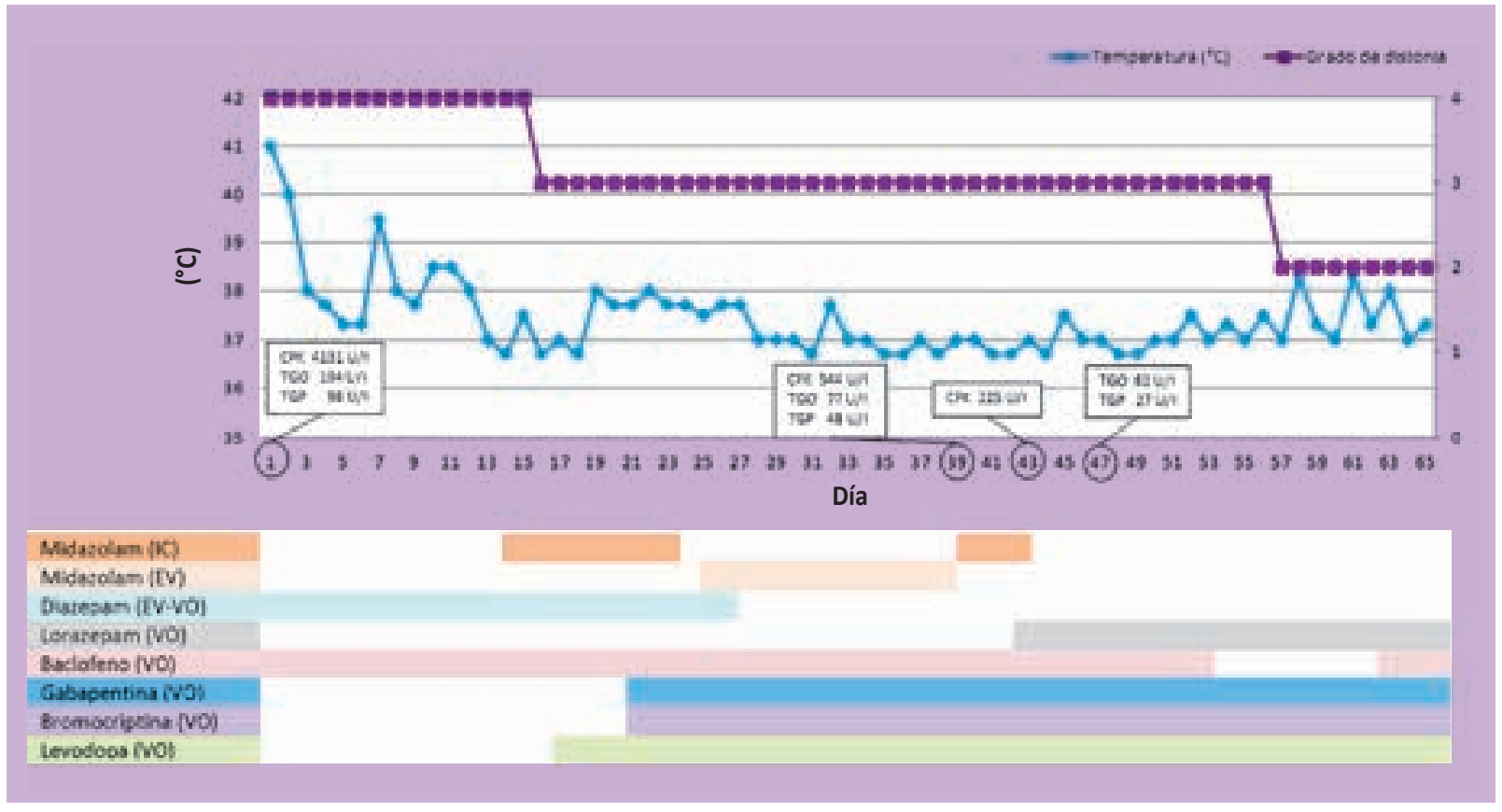

Figura 2. Caso 2, correlación clínica y laboratorial con tratamiento farmacológico instaurado.

la adición de levodopa/carbidopa, gabapentina y bromocriptina. Evoluciona favorablemente al revertir cuadro neumónico, alcanzando un grado 2-ESD al alta. (Figura 2).

\section{DISCUSIÓN}

Desde su primera descripción en 1982, por Jankovic en un niño con distonía generalizada severa, hiperpirexia y mioglobinuria ${ }^{[1,2]}$, el SD ha sido reportado por varios autores, pero carece de una definición precisa y de estudios poblacionales para establecer su real incidencia ${ }^{[7]}$. Fasano y colaboradores, encontraron que en promedio representa el 2,8\% de todos los casos de distonía identificadas, con una tasa de mortalidad que puede llegar al $10 \%{ }^{[3]}$. A nivel latinoamericano son pocas las descripciones en la literatura científica ${ }^{[8,9]}$ y en el Perú no se ha publicado ningún caso a la fecha.

Los pacientes pediátricos con distonía secundaria son los más afectados. Ambos reportes corresponden a niños con distonía secundaria y antecedente de kernícterus. Este escenario es frecuentemente descrito. Se presenta también en distonías primarias $(26 \%)$, heredo-degenerativas $(35 \%)$, pero con más frecuencia en distonías secundarias (38\%), de éstas últimas la $\mathrm{PCl}$ corresponde al 59,3\% ${ }^{[3]}$. Quienes padecieron kernícterus desarrollan parálisis cerebral principalmente distónica, por el daño selectivo de los núcleos basales, sustancia negra y núcleo subtalámico ${ }^{[10]}$, condición que estaría en relación con una mayor susceptibilidad a sufrir cuadros de reagudización distónica.

No solo las infecciones son factores precipitantes de SD. En ambos casos reportados existen antecedentes de episodios de neumonía previa, que no se acompañaron de SD necesariamente.
Si bien las infecciones son el principal factor precipitante en un $51,7 \%$ de los casos ${ }^{[3]}$. Hay otros factores como la adición de fármacos (metoclopramida, haloperidol, pimozide, clonazepam, penicilamina), suspensión de un medicamento regular (baclofeno, tetrabenazina, penicilamina, zolpidem), los traumatismos, procedimientos quirúrgicos, desordenes metabólicos, falla de sistema de estimulación cerebral profunda, reflujo gastroesofágico y constipación ${ }^{[3,11]}$. Todos estos factores deben ser considerados y corregidos.

La rabdomiólisis es frecuentemente descrita en SD. En ambos casos reportados se constató elevación de la CPK-CK y transaminasas sin compromiso hepático, que posteriormente se redujeron en correlación con la mejoría del cuadro clínico (Figura 1 y 2). La elevación del CPK-CK generalmente supera 3 veces el valor normal ${ }^{[9]}$. La transaminasemia, como consecuencia de la destrucción muscular, puede correlacionarse con la severidad de la distonía ${ }^{[12,13]}$, y debería tenerse en cuenta al momento del monitoreo terapéutico y respuesta clínica.

Las benzodiacepinas y la optimización de la terapia previa permiten revertir el SD. En ambos casos se utilizó diazepam y midazolam de forma favorable. Además de la optimización de la terapia previa y adición de levodopa, baclofeno y gabapentina. Es cierto que los anticolinérgicos (trihexifenidilo) o depletores de catecolaminas (tetrabenazina) administrados solos o en combinación muestran una efectividad de $29,2 \%$ incluso superior a las benzodiacepinas ${ }^{[3]}$, pero en el Perú aún no se dispone de estos fármacos. El uso de levodopa, baclofeno, gabapentina ha sido empleado por otros autores, siempre en combinación, obteniéndose resultados diversos ${ }^{[9,14]}$, estos hallazgos ponen en manifiesto que la fisiopatología del SD aún no se conoce por completo, planteándose la posibilidad 
que sean diferentes los mecanismos implicados en su desarrollo ${ }^{[2,15]}$.

En conclusión, el presente trabajo pone en manifiesto la necesidad de identificar los casos de SD, principalmente en pacientes con distonía secundaria, en quienes se debe identificar y corregir los factores gatillantes. Monitorizar los valores de CPK, TGO, TGP y función renal tanto para diagnóstico y evolución. Finalmente se sugiere que ante la sospecha de SD se inicie tratamiento parenteral con benzodiacepinas de acción rápida, ya sea en bolo o infusión continua; paralelamente a la optimización de la terapia oral con baclofeno, levodopa, bromocriptina e incluso gabapentina; hasta que se tenga en el mercado mejores opciones terapéuticas orales como trihexifenidilo o tetrabenazina.

Fuente de financiamiento: autofinanciado.

Conflictos de interés: Los autores declaran no tener conflictos de interés.

\section{REFERENCIAS BIBLIOGRÁFICAS}

1. Jankovic J, Penn AS. Severe dystonia and myoglobinuria. Neurology. 1982;32(10):1195-7.

2. Manji H, Howard RS, Miller DH, Hirsch NP, Carr L, Bhatia K, et al. Status dystonicus: the syndrome and its management. Brain J Neurol. 1998;121(Pt 2):243-52.

3. Fasano A, Ricciardi L, Bentivoglio AR, Canavese C, Zorzi G, Petrovic I, et al. Status dystonicus: predictors of outcome and progression patterns of underlying disease. Mov Disord. 2012;27(6):783-788.

4. Allen NM, Lin J-P, Lynch T, King MD. Status dystonicus: a practice guide. Dev Med Child Neurol. 2014;56(2):105-12.
5. Lumsden DE, Lundy C, Fairhurst C, Lin J-P. Dystonia Severity Action Plan: a simple grading system for medical severity of status dystonicus and life-threatening dystonia. Dev Med Child Neurol. 2013;55(7):671-2.

6. Kaitlin Krebs PH. Status Dystonicus: a rare, but a serious movement disorders emergency. J Neurol Disord [Internet]. 2014 [citado 19 de febrero de 2016];2(6). Disponible en: http://esciencecentral. org/journals/status-dystonicus-a-rare-but-a-serious-movementdisorders-emergency-2329-6895-2-193. php?aid=35624

7. Baxter P. Status dystonicus: under-recognized and under-treated. Dev Med Child Neurol. 2013;55(2):99.

8. Ferrea M, Jorrat, P, Vilariño, J, Intruvini, S, Schteinschnaider, A. Estatus distónico en niños. Reporte de casos y propuesta de tratamiento [Internet]. Revista Neurología Infantil Argentina. 2013;4(5):7-10 [citado 22 de mayo de 2016]. Disponible en: http:// docplayer.es/11328901-Sociedad-argentina-de-neurologia-infantilinfantil-argentina-5ejemplar-numeroneurologia-volumen-4-no5julio-2013-issn-1853-3345-pag.html

9. Teive HAG, Munhoz RP, Souza MM, Antoniuk SA, Santos MLSF, Teixeira MJ, et al. Status Dystonicus: study of five cases. Arq Neuropsiquiatr. 2005;63(1):26-9.

10. Shapiro SM. Chronic bilirubin encephalopathy: diagnosis and outcome. Semin Fetal Neonatal Med. 2010;15(3):157-63.

11. Kojović M, Kramberger MG. Zolpidem withdrawal status dystonicus in the patient with advanced Parkinson's disease. Parkinsonism Relat Disord. 2015;21(6):661-2.

12. Mrkobrada S, Gnanakumar V. The correlation of dystonia severity and serum transaminases in a child with a brain injury. Pediatr Neurol. 2014;51(4):573-5.

13. Muirhead W, Jalloh I, Vloeberghs M. Status dystonicus resembling the intrathecal baclofen withdrawal syndrome: a case report and review of the literature. J Med Case Reports. 2010;4:294.

14. Mariotti P, Fasano A, Contarino MF, Della Marca G, Piastra $\mathrm{M}$, Genovese $\mathrm{O}$, et al. Management of status dystonicus: our experience and review of the literature. Mov Disord Off J Mov Disord Soc. 2007;22(7):963-8.

15. Mishra D, Singhal S, Juneja M. Status dystonicus: A rare complication of dystonia. Indian Pediatr. 2010;47(10):883-885.

\section{Las ediciones anteriores de Acta Médica Peruana están disponibles en:}

\section{www.scielo.org.pe}

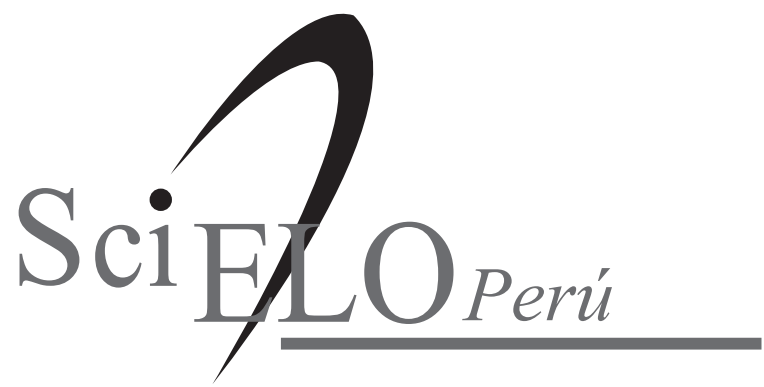

